\title{
SIRT4 Prevents Hypoxia-Induced Apoptosis in H9c2 Cardiomyoblast Cells
}

\author{
Ban Liu ${ }^{a, d}$ Wenliang Che $e^{a, d}$ Jinsong Xue ${ }^{b, d}$ Changzhu Zheng ${ }^{c, d} \quad K a i$ Tang ${ }^{a}$ Jingying \\ Zhang $^{\mathrm{a}}$ Jing Wen ${ }^{\mathrm{a}}$ Yawei $\mathrm{Xu}^{\mathrm{a}}$
}

\begin{abstract}
aDepartment of Cardiology, Shanghai Tenth People's Hospital, Tongji University School of Medicine, Shanghai 200072, P.R. China; bEye hospital, Nanjing Medical University, Nanjing 210029, P.R. China; 'Department of Cardiology, Pudong New Area Zhoupu Hospital, Shanghai 201318, P.R. China; ${ }^{\mathrm{d}}$ contribute equally to this paper as co-first authors
\end{abstract}

\section{Key Words}

SIRT4 • Hypoxia • Apoptosis • Cardiomyoblast

\begin{abstract}
Aims: Apoptosis plays a critical role in cardiomyocyte loss during ischaemic heart injury. A detailed understanding of the mechanism involved has a substantial impact on the optimization and development of treatment strategies. Here, we report that the expression of SIRT4, a mitochondrial sirtuin, is markedly down-regulated in hypoxia-induced apoptosis of H9c2 cardiomyoblast cells. Methods and Results: SIRT4 interference significantly alters H9c2 cell viability, apoptotic cell number and caspase-3/7 activity. Furthermore, SIRT4 expression can affect the ratio of pro-caspase 9/caspase 9 or pro-caspase 3/caspase 3, an affect Bax translocation, which in turn alters the development of $\mathrm{H} 9 \mathrm{c} 2$ cell apoptosis. Conclusion: These results suggest that SIRT4 is a key player in hypoxia-induced cardiomyocyte apoptosis, and that strategies based on its enhancement might be of benefit in the treatment of ischaemic heart disease.
\end{abstract}

\section{Introduction}

Cardiovascular disease is becoming a major cause of death, accounting for more than one third of global morbidity. The adult cardiomyocyte is not capable of cell proliferation [1]. In fact, significant loss of cardiomyocytes by apoptosis is an important pathogenic feature of a variety of cardiovascular diseases, including heart failure, myocardial ischemia and infarction [2]. 
Hypoxia is able to affect the extent of cell injury and death during acute and chronic myocardial ischemia and infarction [3]. It triggers mitochondrial permeability, and triggers apoptosis in cardiomyocytes, endothelial cells and vascular smooth muscle cells. Increasing evidences have shown that hypoxia results in cell apoptosis, through a mitochondrial dependent process involving release of cytochrome c, activation of caspase 9 and subsequent cleavage and activation of downstream, effector caspases [4, 5], suggesting that mitochondria plays a central role in the regulation of hypoxia-induced apoptosis.

Sirtuins are NAD-dependent deactylases that have homology to the yeast Sir2 protein. To date, seven homologs are reported expressed in mammalian cell. SIRT1 localizes primarily in the nucleus along with SIRT6 and SIRT7. SIRT2 is in the cytoplasm whereas SIRT3, SIRT4, and SIRT5 are localized in the mitochondria [6, 7]. Of these mitochondrial sirtuins, SIRT4 is found to be specifically enriched in the heart, kidney, brain, and liver. SIRT4 does not have $\mathrm{NAD}^{+}$-dependent deacetylase activity, which is significantly different from other sirtuins, but functions as an efficient ADP-ribosyltransferase on histones and bovine serum albumin [8]. SIRT4 has been implicated in the regulation of insulin secretion by modulation of glutamate dehydrogenase. Furthermore, SIRT4 inhibition increases fat oxidative capacity in liver and mitochondrial function in muscle [9-11]. There evidences suggest that SIRT4 level is tightly associated with metabolism status. Significant change in SIRT4 expression could affect cell viability, even result in cellular apoptosis. To date, there is still no report about the direct role of SIRT4 in hypoxia-induced cardiomyocyte apoptosis.

In the present study, therefore, we investigated the role of SIRT4 in the survival of H9c2 cardiomyoblast cells against hypoxia stimulus as well as the underlying mechanism. We found that SIRT4 exerts cardioprotection against hypoxia-induced apoptosis. The inhibition of caspase activation and Bax translocation plays an important role in mediating the antiapoptotic effect of SIRT4.

\section{Materials and Methods}

\section{Cell culture and transfection}

H9c2 cells were cultured in Dulbecco's modified Eagle's medium (DMEM) with 10\% calf serum. For SIRT4 silencing experiment, the transfection of $\mathrm{H} 9 \mathrm{c} 2$ cells were done using four siRNA duplexes directed at different regions of SIRT4 or scrambled siRNA pool purchased from Dharmacon (Lafayette, CO). For SIRT4 overexpression experiment, H9c2 cells were transfected with SIRT4 plasmid or vector plasmid. All transient transfection was performed by using lipofectamine 2000 according to the manufacturer's protocol (Invitrogen). H9c2 cells were incubated in DMEM medium without antibiotics for $24 \mathrm{~h}$ before transfection to enhance the transfection efficiency.

\section{Establishment of hypoxic culture condition}

H9c2 cells were grown to the desired confluence and then transferred to an air tight Plexiglas hypoxic chamber in glucose-free medium containing 2\% Oxyrase. Establishment of environmental hypoxic conditions $(<1 \%)$ was achieved by continuously flushing the chamber with a water saturated mixture of 5\% $\mathrm{CO}_{2}$ and $95 \% \mathrm{~N}_{2}$. Maintenance of the desired $\mathrm{O}_{2}$ concentration was constantly monitored during incubation using a microprocessor-based oxygen sensor.

\section{Real-time PCR analysis}

The relative levels of SIRT4 mRNA were quantified by using real-time PCR. Briefly, the converted cDNA samples $(2 \mu \mathrm{l}$ ) were amplified in a final volume of $25 \mu \mathrm{l}$ using SYBR Green Master Mix reagent (Applied Biosystems) in the ABI Prism 7500 sequence detection system. Melting curve analysis was performed using Dissociation Curves soft-ware (Applied Biosystems) to ensure that only a single product was amplified. The specificity of the reactions was confirmed by $2 \%$ agarose gel electrophoresis. Results were obtained using ABI Prism sequence detection software and evaluated using Excel (Microsoft). 
Liu et al.: SIRT4 Induced Apoptosis in H9c2 Cells

\section{Western blot}

Cells were lysed with a lysis buffer $\left(50 \mathrm{mM}\right.$ Tris, $50 \mathrm{mM} \mathrm{KCl,} 20 \mathrm{mM} \mathrm{NaF}, 1 \mathrm{mM} \mathrm{Na}_{3} \mathrm{VO}_{4}, 10$ mM ethylenediaminetetraacetic acid (EDTA), 1\% NP-40, $10 \mathrm{mM}$ nicotinamide, $1 \mathrm{mM}$ TSA, $1 \mathrm{mM}$ phenylmethanesulphonylfluoride (PMSF), $5 \mu \mathrm{g} \mathrm{m}^{-1}$ leupeptin, $\mathrm{pH}$ 8.0). Western blot experiments were done after the specific treatment and sample collection. Cell lysate was fractionated by SDS-10\% polyacrylamide gel electrophoresis and transferred to PVDF membranes (Amersham). After blocking with recommended blocking reagents for $1 \mathrm{~h}$ at the room temperature, the membranes were incubated overnight at $4^{\circ} \mathrm{C}$ with different antibodies, and then these membranes were incubated with 1:5000-1:10000 secondary antibodies conjugated with HRP. Signals were detected by using the Amersham ECL chemiluminescence system.

\section{Analysis of cell viability}

The 3-(4, 5-dimethylthiazal-2-yl)-2, 5-diphenyl-tetrazolium bromide (MTT) assay was used to estimate cell viability $[12,13]$. Briefly, cells were plated at a density of $1 \times 10^{4}$ cells per well in 96 -well plates. After exposure to specific treatment, the cells were incubated with MTT at a final concentration of $0.5 \mathrm{mg} / \mathrm{ml}$ for $3 \mathrm{~h}$ at $37^{\circ} \mathrm{C}$. After the removal of the medium, $100 \mathrm{mM}$ DMSO solutions were added to dissolve the formazan crystals. The absorbance at $570 \mathrm{~nm}$ wavelength was detected using a microplate reader (Synergy 4 Hybrid Multi-Mode; BioTek Instruments).

Detection of apoptosis percentage and caspase 3/7 activity

Apoptosis was induced by hypoxia treatment. Cells were stained with DAPI and the apoptotic nuclei were counted under a fluorescence microscope (500 cells were counted for each experiment). Caspase 3/7 activity was assayed by Promega kit according to the manufacturer's protocol. Caspase-Glo 3/7 Reagent (Promega) was added to each well in a 1:1 ratio following manufacturer's instructions. After 10 min on a plate shaker at room temperature, $90 \%$ of the lysate volume was transferred to a 96-well solid-white plate. Cell lysates were analyzed by using a microplate reader (Synergy 4 Hybrid Multi-Mode; BioTek Instruments), and data points were blank subtracted. Assays were performed in triplicate and are reported as mean \pm S.E.M. [14, 15].

\section{Subcellular fractionation}

Mitochondrial and cytosol cellular fractions were prepared using a Cytosol/Mitochondria Fractionation kit (Cabiochem). H9c2 cells were harvested by centrifugation at $1,000 \mathrm{~g}$ for $10 \mathrm{~min}$ at $4^{\circ} \mathrm{C}$ and washed twice with cold PBS. Afterward, H9c2 cells were resuspended in $250 \mu \mathrm{l}$ Cytosol Extraction buffer containing a protease inhibitor cocktail and $1 \mathrm{mM}$ dithiothreitol (DTT). After incubation on ice for $10 \mathrm{~min}, \mathrm{H} 9 \mathrm{c} 2$ cells were homogenized on ice using a dounce tissue homogenizer. Homogenized cells were centrifuged at 1,000 $g$ for $15 \mathrm{~min}$ at $4^{\circ} \mathrm{C}$, and supernatants were collected. Supernatants were then centrifuged again at $10,000 \mathrm{~g}$ for $30 \mathrm{~min}$ at $4^{\circ} \mathrm{C}$. The resulting supernatants were harvested and designated as cytosolic fractions and the pellets were resuspended in $50 \mu \mathrm{l}$ Mitochondria Extraction buffer containing a protease inhibitor cocktail and $1 \mathrm{mM}$ DTT and designated as mitochondrial fractions. All fractions were stored at $-80^{\circ} \mathrm{C}$ until use.

\section{Statistical analysis}

All values were expressed as the mean \pm S.E.M. Comparisons between 2 groups were conducted by using one-way analysis of variance. Post hoc analysis was performed by Fisher's protected least significant difference test. $P<0.05$ were considered significant.

\section{Result}

Aberrant SIRT4 expression in hypoxia-induced apoptosis in H9c2 cell

To detect the expression pattern of SIRT4 in hypoxia-induced apoptosis in H9c2 cell, we employed RT-PCR method to compare the expression difference of SIRT4 mRNA between the hypoxia-treated group and the matched normal group. As shown in Fig. 1A, the expression of SIRT4 mRNA appeared to be markedly down-regulated in the hypoxia-treated group compared to the matched normal group. Meanwhile, western blot analysis revealed that the expression of SIRT4 level was markedly down-regulated in hypoxia-treated group at protein 
Fig. 1. Aberrant expression of SIRT4 in hypoxia-induced H9c2 cell apoptosis. (A)H9c2 cells were subjected to normoxia or hypoxia for $12 \mathrm{~h}, 16$ h or, 24 h. SIRT4 mRNA expression was detected by real-time PCR. Each sample was analyzed in triplicate. Asterisk indicates significant difference compared with the normoxia group $(P<0.05)$. (B) H9c2 cells were treated as shown in Fig.1A. SIRT4 protein expression in whole cell lysates of H9c2 cells was detected by western blots. Shown is a representative image

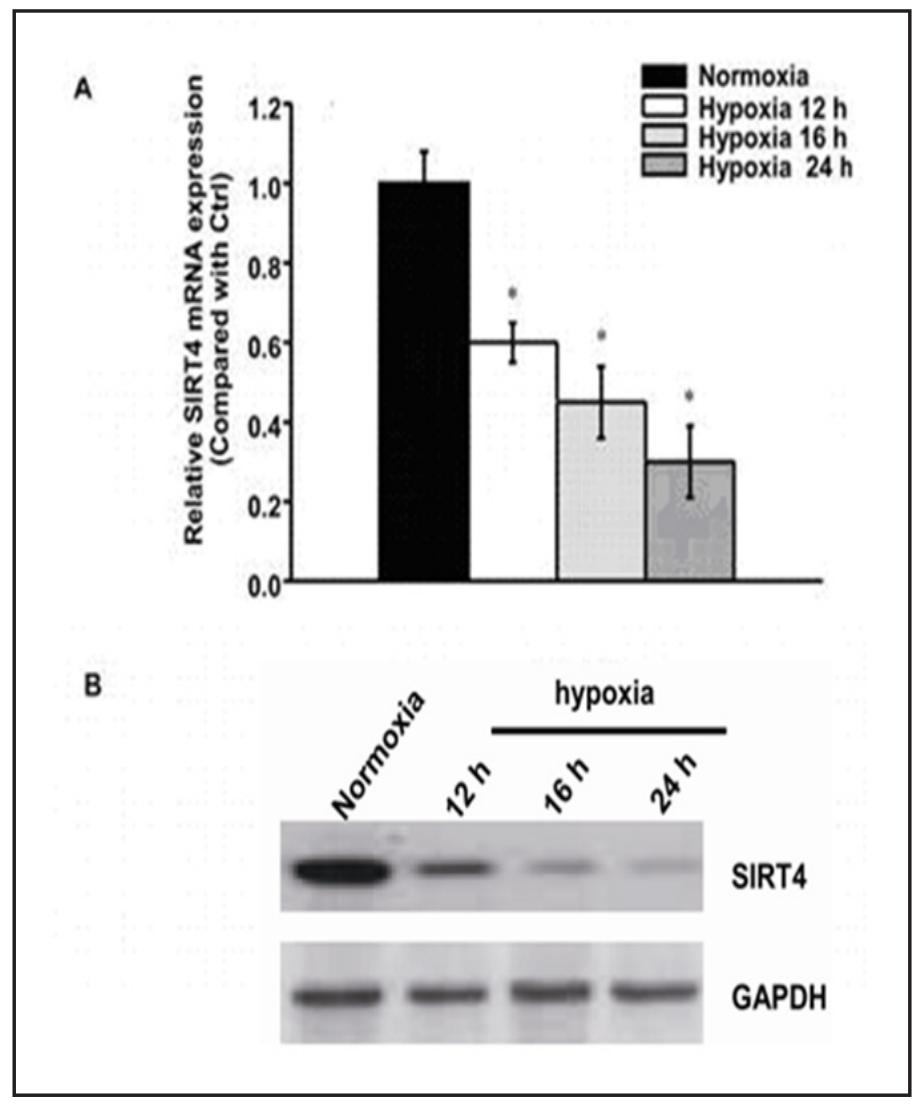

level (Fig. 1B). Hence, these results indicated that the expression of SIRT4 was repressed during hypoxia-induced apoptosis in a time-dependent manner.

SIRT4 interference affects hypoxia-induced apoptosis in H9c2 cells

To determine the role of SIRT4 in hypoxia-induced apoptosis in H9c2 cells, we conducted SIRT4 over-expression or SIRT4 knockdown experiment to alter SIRT4 level. Western blots analysis showed that SIRT4 level was effectively down-regulated by the transfection of SIRT4 siRNA, and significantly enhanced by SIRT4 overexpression (Fig. 2A). Compared with the control group, SIRT4 overexpression could significantly increase H9c2 cell viability, and significantly decrease the percentage of apoptotic cells and the activity of caspase-3/7 in response to hypoxia treatment. By contrast, SIRT4 knockdown via treatment with SIRT4 siRNA exhibited the opposite phenotype (Fig. 2B-D). Taken together, these results suggest that SIRT4 plays a protective role in hypoxia-induced apoptosis in H9c2 cells.

Effect of SIRT4 interference on caspase expression during hypoxia-induced apoptosis

The mitochondria act at the core of the apoptotic pathway by providing many important factors that induce caspase activation and chromosome fragmentation. Released cytochrome $c$, Apaf1, and procaspase-9 from the mitochondria interact with each other to form the apoptosome that drives the activation of caspase 3 [16]. Thus, we detected the expression of caspase 3 and caspase- 9 due to their crucial role in regulating apoptotic process. We found that hypoxia treatment resulted in a significant increase in the amount of the cleaved caspase 3 or caspase 9. Further, SIRT4 silencing treatment could further increase the amount of cleaved caspase 3 or caspase 9 . By contrast, SIRT4 overexpression significantly reduced the amount of cleaved caspases 3 and 9 caused by hypoxia treatment (Fig. 3A and 3B). Thus, these evidences suggest that SIRT4 could exert its regulatory effect on H9c2 cell apoptosis through regulating the ratio of pro-caspase $9 /$ caspase 9 and pro-caspase $3 /$ caspase 3 . 


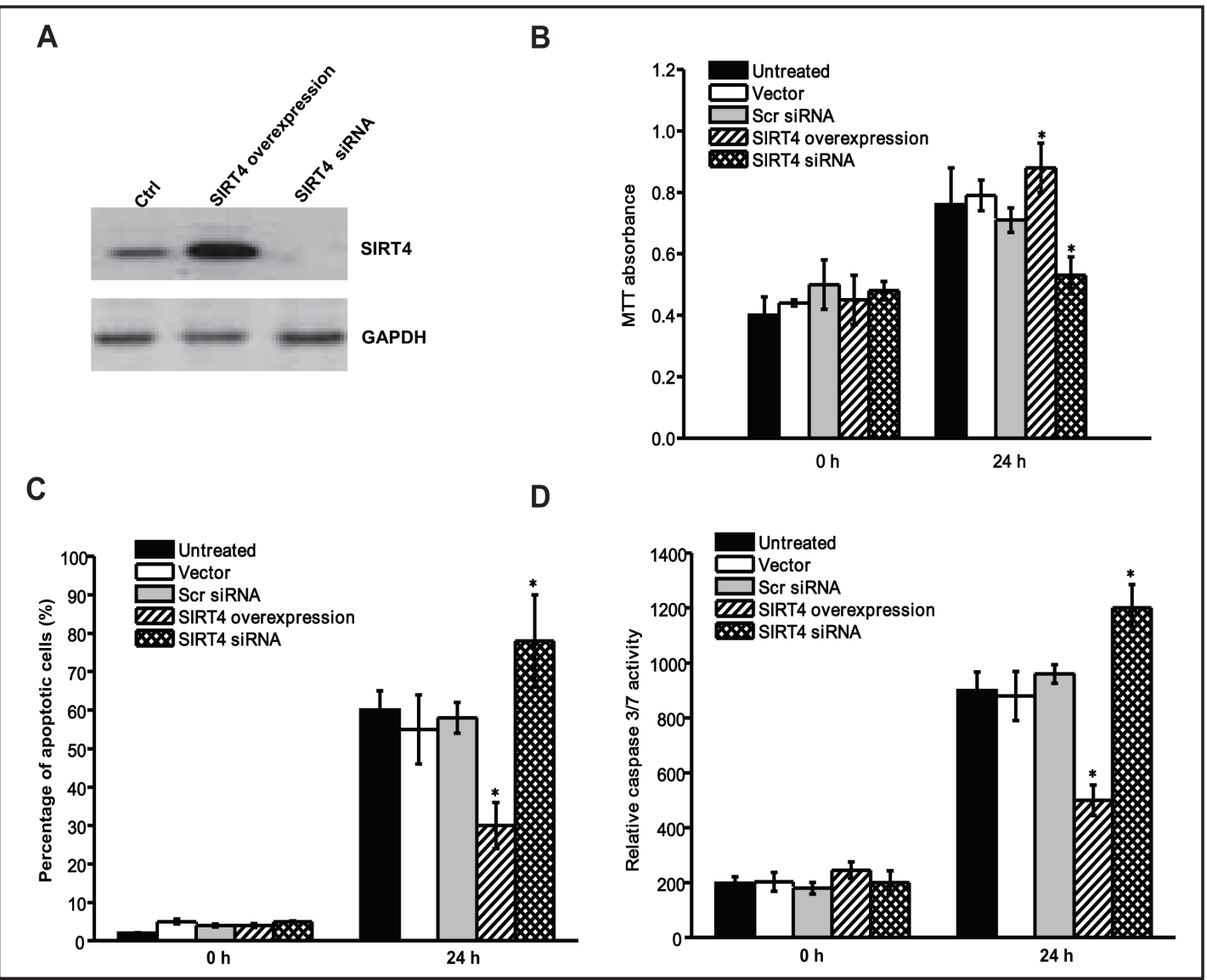

Fig. 2. SIRT4 interference could affect the development of hypoxia-induced apoptosis in H9c2 cells. (A) H9c2 cells were transfeced with SIRT4, SIRT4 siRNA, scramble siRNA, vector, or left untreated, and then exposed to hypoxia condition for $24 \mathrm{~h}$. The expression of SIRT4 level was detected by Western blot. GAPDH was detected as the loading control. Shown is a representative image. (B-D) H9c2 cells were treated as shown in Fig. 2A, and then H9c2 cells were exposed to hypoxia to induce cell apoptosis for $24 \mathrm{~h}$. These untreated cells were used as the control group. Cell viability was determined using MTT assay kit (B). The nuclei were stained with DAPI, and apoptotic nuclei were counted under a fluorescence microscope (300 cells were counted for each experiment) (C). The activity of caspase 3/7 was determined using caspase 3/7 assay kit (Promega) as described in "Materials and methods" section (D). Results are expressed as means \pm S.E.M. of three independent experiments. Each sample was analyzed in triplicate $(P<0.05)$. Asterisk indicates significant difference compared with the untreated group.

\section{Modulation of Bax translocation by SIRT4 in hypoxia-induced apoptosis}

The Bcl-2 family regulates the mitochondrial pathway of apoptosis by controlling the permeabilization of the outer mitochondrial membrane. Among Bcl-2 family, Bax and Bcl2 are recognized as two of the most important members that exerts either pro- or antiapoptotic effects in cells $[17,18]$. We found that SIRT4 level does not change the total amount of Bax and Bcl-2 protein extracted from H9c2 cells (Fig. 4A). SIRT4 expression level does not change the mitochondrial or cytoplasm fraction of Bax and Bcl-2 protein. In response to hypoxia stress, the translocation of Bax to mitochondria was also studied in the H9c2 cells expressed different levels of SIRT4 protein. As shown in Fig. 4B, translocation of Bax to mitochondria was obvious in SIRT4 silencing H9c2 cells. By contrast, Bax translocation to mitochondria was significantly reduced in SIRT4 overexpression cells. Meanwhile, we also detected the cytoplasm fraction of Bax amount by using western blots. We found that Bax amount in the cytoplasm fraction of SIRT4 silencing cells was significantly lower than that 
Fig. 3. Effect of SIRT4 interference on caspase 3/9 expression in hypoxiainduced apoptosis in $\mathrm{H} 9 \mathrm{c} 2$ cells. (A and B) H9c2 cells were transfeced with SIRT4, SIRT4 siRNA or left untreated, and then exposed to hypoxia for $24 \mathrm{~h}$. The expression of full length or cleaved caspase 3/9 was detected by Western blot. GAPDH was detected as the loading control. Shown is a representative image.
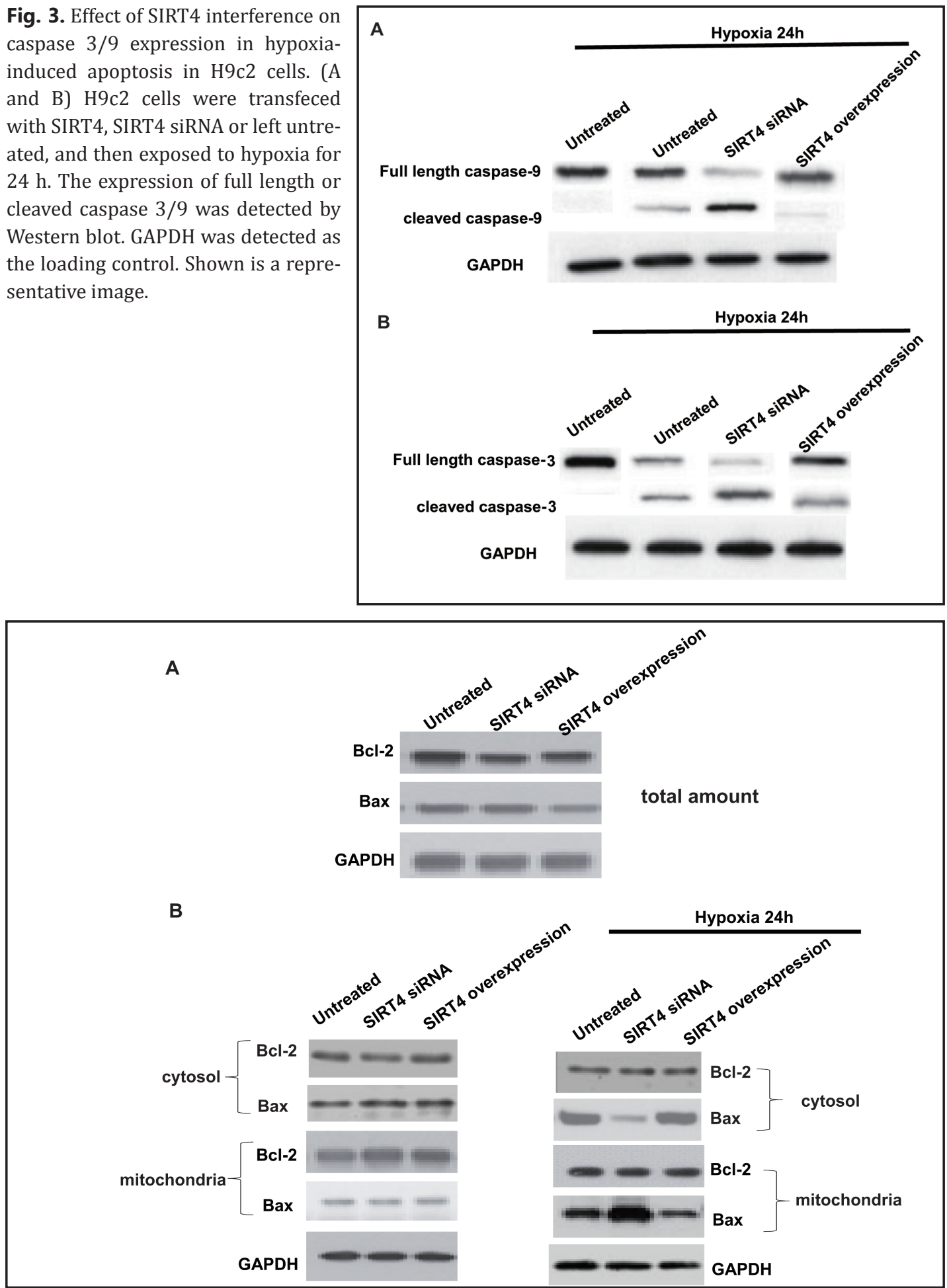

Fig. 4. Modulation of Bax translocation by SIRT4 in hypoxia-induced apoptosis. (A) H9c2 cells were transfeced with SIRT4, SIRT4 siRNA or left untreated. Western blots were performed to detect the total amount of Bax and Bcl-2 expression in H9c2 cells. Results are representative of those from 3 independent experiments. (B) H9c2 cells were treated as shown in Fig. 4A, and then were exposed to hypoxia for $24 \mathrm{~h}$. The group without hypoxia treatment was taken as the control group. Western blots were performed to detect the mount of Bax and Bcl-2 in the mitochondrial fraction and the cytoplasm fraction. Results are representative of those from 3 independent experiments. 
in SIRT4 overexpression cells. Taken together, these results suggest that SIRT4 regulates the apoptosis of $\mathrm{H} 9 \mathrm{c} 2$ cells through affecting Bax translocation.

\section{Discussion}

Apoptosis plays a critical role in the pathogenesis of many cardiovascular diseases including atherosclerosis, myocardial ischemia and reperfusion injury, diabetic cardiomyopathy, and chronic heart failure [19]. A variety of key events in apoptosis is tightly associated with mitochondria, including the release of caspase activators (such as cytochrome $c$ ), alteration in electron transport, loss of mitochondrial transmembrane potential, and participation of pro- and anti-apoptotic Bcl-2 family proteins [20]. These different signals converge on mitochondria, and eventually change the development of cellar apoptosis. Here, we identified a novel mitochondrial regulator of apoptosis, SIRT4, which plays a key role in hypoxia-induced apoptosis in H9c2 cardiomyoblast cells.

Mitochondria is involved in apoptosis by the activation of mitochondrial outer membrane permeabilization [21]. Outer membrane permeabilization leads to caspase activation. Caspases are a family of aspartate-specific cysteine proteases responsible for the biochemical and morphological changes that occur during the execution phase of apoptosis [22]. Caspase-9 is the apical caspase mitochondrial apoptosis pathways. It can directly processes and activates the effector caspase, caspase-3, which is a major effector caspase responsible for the cleavage of cellular substrates during apoptosis [23]. Here, we found that hypoxia treatment results in a significant increase in cleaved caspase 3 or caspase 9. SIRT4 interference could significantly change the amount of the cleaved and the full length caspase $3 / 9$. These results suggest that SIRT4 could regulate the ratio of pro-caspase $9 /$ caspase 9 and pro-caspase 3/caspase 3, which in turn affect the development of $\mathrm{H} 9 \mathrm{c} 2$ cell apoptosis.

The subcellular localization and translocation of Bcl-2 family members are the key players in the process of cellular apoptosis [24]. Bax has been found in the cytoplasm or loosely attached to mitochondria as a monomeric protein under normal conditions [21, 25]. Upon apoptotic stimulation, Bax undergoes a conformational change and translocates to mitochondria via the mitochondrial targeting sequence at the $C$ terminus. A number of proteins have been proposed to bind to and regulate Bax translocation. Truncated Bid (tBid) , PUMA and some non-Bcl-2 family proteins, such as Bif-1 and p53, have been found to important regulators of Bax translocation $[15,26]$. In this study, we found that SIRT4 could affect Bax translocation. Upon hypoxia stimulus, there is a significant increase in the level of mitochondrial Bax. SIRT4 level could significantly affect the degree of mitochondrial Bax protein, suggesting that Bax translocation is critical step in the development of SIRT4mediated cellular apoptosis.

In summary, SIRT4, a mitochondrial sirtuin, is identified as an important regulator of cellular apoptosis. Its expression is markedly reduced in hypoxia-induced apoptosis in H9c2 cardiomyoblast cells. SIRT4 interference could significantly alter H9c2 cell viability, and affect the apoptotic cell number and caspase-3/7 activity. Furthermore, SIRT4 expression could affect the ratio of pro-caspase $9 /$ caspase 9 or pro-caspase $3 /$ caspase 3 , and change Bax translocation, which in turn alter the development of H9c2 cell apoptosis. Thus, Sirt4 acts as an important regulator of H9c2 cell apoptosis. SIRT4 intervene would provide a novel strategy for the treatment of cardiovascular disease caused by hypoxia-induced apoptosis.

\section{Acknowledgements}

This work was supported by National Natural Science Foundation of China (Grant No. 81200131 to B.L.). 
Liu et al.: SIRT4 Induced Apoptosis in H9c2 Cells

\section{Reference}

1 Taki J, Higuchi H, Kawashima A, Tait JF, Kinuya S, Muramori A, Matsunari I, Nakajima K, Tonami N, Strauss HW: Detection of cardiomyocyte death in a rat model of ischemia and reperfusion using $99 \mathrm{mTc}$-labeled annexin V. J Nucl Med 2004;45:1536-1541.

2 Marín-García J, Akhmedov AT, Moe GW: Mitochondria in heart failure: The emerging role of mitochondrial dynamics. Heart Fail Rev 2013;18:439-56. .

- 3 Graham RM, Frazier DP, Thompson JW, Haliko S, Li H, Wasserlauf BG, Spiga MG, Bishopric NH, Webster KA: A unique pathway of cardiac myocyte death caused by hypoxia-acidosis. J Exp Biol 2004;207:3189-3200.

4 Pop C, Salvesen GS: Human caspases: activation, specificity, and regulation. J Biol Chem 2009;284:2177721781.

5 Inoue S, Browne G, Melino G, Cohen G: Ordering of caspases in cells undergoing apoptosis by the intrinsic pathway. Cell Death Differ 2009;16:1053-1061.

6 Imai SI, Guarente L: Ten years of NAD-dependent SIR2 family deacetylases: implications for metabolic diseases. Trends Pharmacol Sci 2010;31:212-220.

7 Verdin E, Hirschey MD, Finley LW, Haigis MC: Sirtuin regulation of mitochondria: energy production, apoptosis, and signaling. Trends Biochem Sci 2010;35:669-6751.

8 Mahlknecht U, Voelter-Mahlknecht S: Fluorescence in situ hybridization and chromosomal organization of the sirtuin 4 gene ( Sirt4) in the mouse. Biochem Bioph Res Co 2009;382:685-690.

-9 Nasrin N, Wu X, Fortier E, Feng Y, Bare OC, Chen S, Ren X, Wu Z, Streeper RS, Bordone L: SIRT4 regulates fatty acid oxidation and mitochondrial gene expression in liver and muscle cells. Sci Signal 2010;285:31995.

10 Haigis MC, Mostoslavsky R, Haigis KM, Fahie K, Christodoulou DC, Murphy AJ, Valenzuela DM, Yancopoulos GD, Karow M, Blander G: SIRT4 inhibits glutamate dehydrogenase and opposes the effects of calorie restriction in pancreatic $\beta$ cells. Cell 2006;126:941-954.

11 Ahuja N, Schwer B, Carobbio S, Waltregny D, North BJ, Castronovo V, Maechler P, Verdin E: Regulation of insulin secretion by SIRT4, a mitochondrial ADP-ribosyltransferase. J Biol Chem 2007;282:33583-33592.

12 Zhang Z, Cao X, Xiong N, Wang H, Huang J, Sun S, Liang Z, Wang T: DNA polymerase- $\beta$ is required for 1-methyl-4-phenylpyridinium-induced apoptotic death in neurons. Apoptosis 2010;15:105-115.

13 Yan B, Zhao JI: miR-1228 prevents cellular apoptosis through targeting of MOAP1 protein. Apoptosis 2012;17:717-724.

14 Najjar NEI, Chatila M, Moukadem H, Vuorela H, Ocker M, Gandesiri M, Schneider-Stock R, Gali-Muhtasib H: Reactive oxygen species mediate thymoquinone-induced apoptosis and activate ERK and JNK signaling. Apoptosis 2010;15:183-195.

15 Yan B, Kong M, Chen YH: Prevention of apoptosis by the interaction between FIH1 and Bax. Mol Cell Biochem 2011;348:1-9.

16 Yuan S, Yu X, Asara JM, Heuser JE, Ludtke SJ, Akey CW: The holo-apoptosome: activation of procaspase-9 and interactions with caspase-3. Structure 2011;19:1084-1096.

17 Kang MH, Reynolds CP: Bcl-2 inhibitors: targeting mitochondrial apoptotic pathways in cancer therapy. Clin Cancer Res 2009;15:1126-1132.

18 Levine B, Sinha SC, Kroemer G: Bcl-2 family members: dual regulators of apoptosis and autophagy. Autophagy 2008;4:600-606.

19 Lee Y, Gustafsson ÅB: Role of apoptosis in cardiovascular disease. Apoptosis 2009;14:536-548.

20 Chen SD, Yang DI, Lin TK, Shaw FZ, Liou CW, Chuang YC: Roles of oxidative stress, apoptosis, PGC-1 $\alpha$ and mitochondrial biogenesis in cerebral ischemia. Int J Mol Sci 2011;12:7199-7215.

21 Martinou JC, Youle RJ: Mitochondria in apoptosis: Bcl-2 family members and mitochondrial dynamics. Dev Cell 2011;21:92-101.

22 Chipuk JE, Green DR: How do BCL-2 proteins induce mitochondrial outer membrane permeabilization? Trends Cell Biol 2008;18:157-164.

23 Wang C, Youle RJ: The role of mitochondria in apoptosis*. Annu Rev Genet 2009;43:95-118.

24 Brunelle JK, Letai A: Control of mitochondrial apoptosis by the Bcl-2 family. J Cell Sci 2009;122:437-441.

25 Kushnareva Y, Andreyev AY, Kuwana T, Newmeyer DD: Bax activation initiates the assembly of a multimeric catalyst that facilitates Bax pore formation in mitochondrial outer membranes. PLoS Biol 2012;10:e1001394.

26 Kim H, Tu HC, Ren D, Takeuchi O, Jeffers JR, Zambetti GP, Hsieh JJ, Cheng EH: Stepwise activation of BAX and BAK by tBID, BIM, and PUMA initiates mitochondrial apoptosis. Mol Cell 2009;36:487-499. 